\title{
Primary Lung Adenocarcinoma With Trophoblastic Differentiation and Usage of Gefitinib for Postoperative Recurrence: Report of a Case With a Review of the Literature
}

\author{
Yasumichi Yamamoto $^{\mathrm{a}, \mathrm{b}}$, Toshiya Toyazaki ${ }^{\mathrm{a}}$, Shinji Kosaka ${ }^{\mathrm{a}}$
}

\begin{abstract}
We report a rare and very aggressive case of lung carcinoma showing trophoblastic differentiation, so-called choriocarcinoma, for which postoperative recurrence was treated using gefitinib. A 51-year-old woman with pathological stage IIA adenocarcinoma with trophoblastic differentiation underwent left lower lobectomy with lymph node dissection and postoperative adjuvant chemotherapy, remaining tumor-free for 10 months until the detection of the brain metastasis. Gefitinib was administered, as the tumor was positive for epidermal growth factor receptor mutation, and this resulted in a 12-month progression-free period. The patient died 37 months after surgery due to multiple metastases in the brain, ovary and uterus. This is the first case report using gefitinib to the lung carcinoma showing trophoblastic differentiation. A review of the literature on lung cancer with trophoblastic differentiation suggested that a chemotherapeutic regimen for primary lung cancer other than germ cell tumor might be suitable for the lung carcinoma with trophoblastic differentiation.
\end{abstract}

Keywords: Lung cancer; Adenocarcinoma; Trophoblastic differentiation; Gefitinib; Choriocarcinoma

\section{Introduction}

We report a case of the lung carcinoma with trophoblastic differentiation so-called choriocarcinoma of the lung, of which the postoperative recurrence was treated by gefitinib. This carcinoma was very aggressive and we had difficulty selecting the chemotherapeutic agents for the treatment of the postoperative recurrences, therefore, we reviewed the literatures concerning the chemotherapeutic regimen of the lung carcinoma

\footnotetext{
Manuscript accepted for publication October 10, 2016

aDepartment of Thoracic Surgery, Shimane Prefectural Central Hospital, Shimane, Japan

${ }^{b}$ Corresponding Authors: Yasumichi Yamamoto, Department of Thoracic Surgery, Shimane Prefectural Central Hospital, 4-1-1 Himebara, Izumo, Shimane 693-8555, Japan. Email: yyama@spch.izumo.shimane.jp
}

doi: http://dx.doi.org/10.14740/jmc2662w with trophoblastic differentiation or the choriocarcinoma of the lung.

\section{Case Report}

A 51-year-old woman visited us because of a nodular shadow in the left lung without any subjective symptoms. Physical examination was normal. She had never smoked and had no history of abnormal pregnancy or abortion. Laboratory examinations demonstrated elevation of the serum level of carcinoembryonic antigen (CEA) at $31.1 \mathrm{ng} / \mathrm{mL}$ and SLX at 45.8 $\mathrm{U} / \mathrm{mL}$. Chest X-ray and computed tomography (CT) demonstrated a nodule with spicula and pleural indentation in the left S6 without lymphadenopathy (Fig. 1a, b). CT-guided needle biopsy revealed poorly differentiated adenocarcinoma with partial trophoblastic differentiation. Gynecological examination including magnetic resonance imaging (MRI) revealed no abnormality except for a uterine leiomyoma and an elevated serum level of hCG at $4.9 \mathrm{mIU} / \mathrm{mL}$ (SV 1.0). No distant metastasis was detected by abdominal CT, brain MRI and bone scintigraphy. The clinical stage of this tumor was cT1bN0M0 stage IA.

Left lower lobectomy and lymph node dissection was performed in December 2009. Pathological examination revealed that the tumor was $26 \times 24 \mathrm{~mm}$ in size without pleural invasion and the interlobular lymph node was involved. Therefore, the postoperative staging was revised to $\mathrm{pT} 1 \mathrm{bN} 1 \mathrm{M} 0$ stage IIA. Light microscopy revealed that the tumor had two different histological components: mixed adenocarcinoma showing solid with mucinous or acinar adenocarcinoma, and trophoblastic differentiation with syncytiotrophoblast-like multinucleated giant cells (Fig. 2a, b). Immunohistological examination demonstrated diffuse positivity for CK7, CEA, IMP-3, Ki67 and TTF-1, but negativity for CK20. The hCG-positive cells were scattered in both the area of adenocarcinoma and trophoblastic differentiation.

Postoperative adjuvant chemotherapy was administrated with four cycles of carboplatin and paclitaxel. The serum level of hCG decreased to $3.4 \mathrm{mIU} / \mathrm{mL}$ being the minimum value throughout the observation period. In October 2010, brain MRI revealed three metastases when the serum level of hCG had increased to $5.0 \mathrm{mIU} / \mathrm{mL}$, and gamma-knife therapy was 

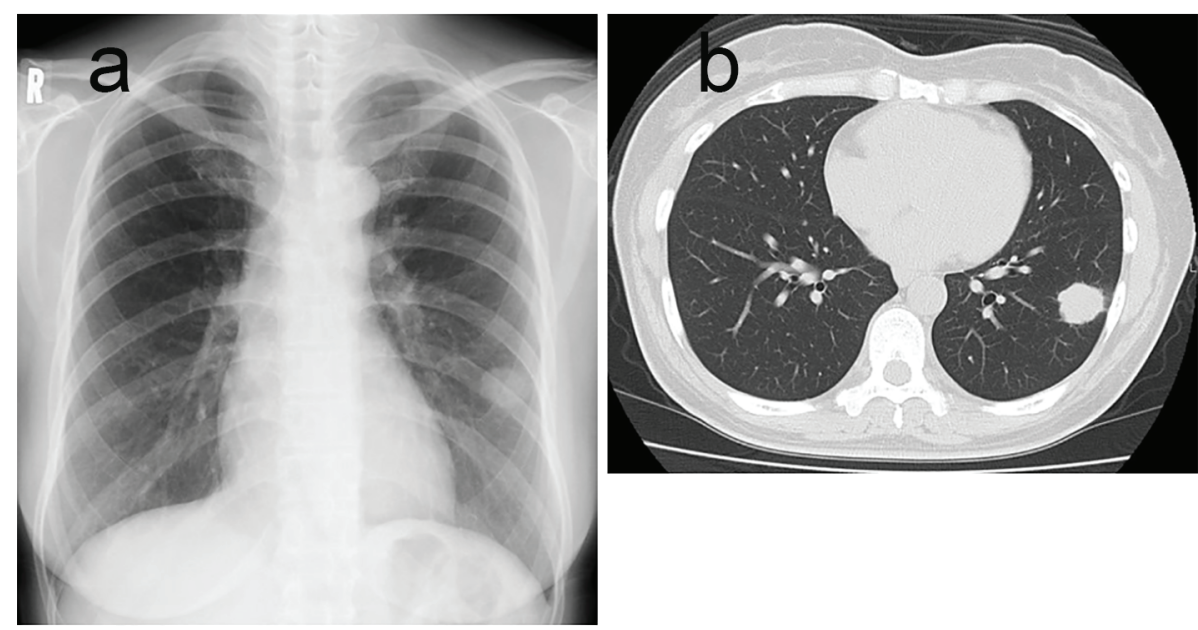

Figure 1. Preoperative chest X-ray showing a $2.5-\mathrm{cm}$ round shadow in the left lung (a). CT revealed spicula and pleural indentation in S6 (b).
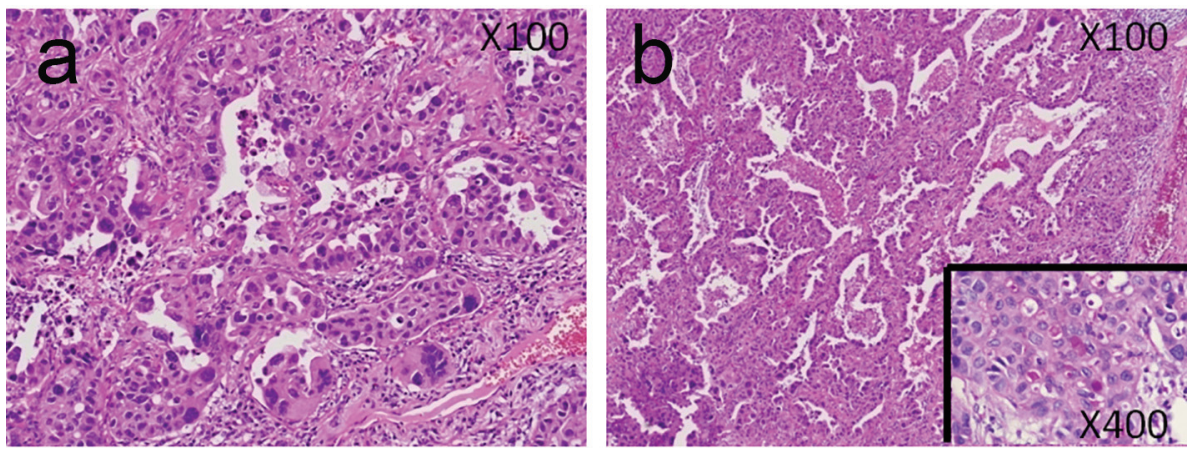

Figure 2. Pathological examination demonstrated mixed adenocarcinoma with trophoblastic differentiation (a), and acinar-type non-mucous bronchioloalveolar carcinoma (b).
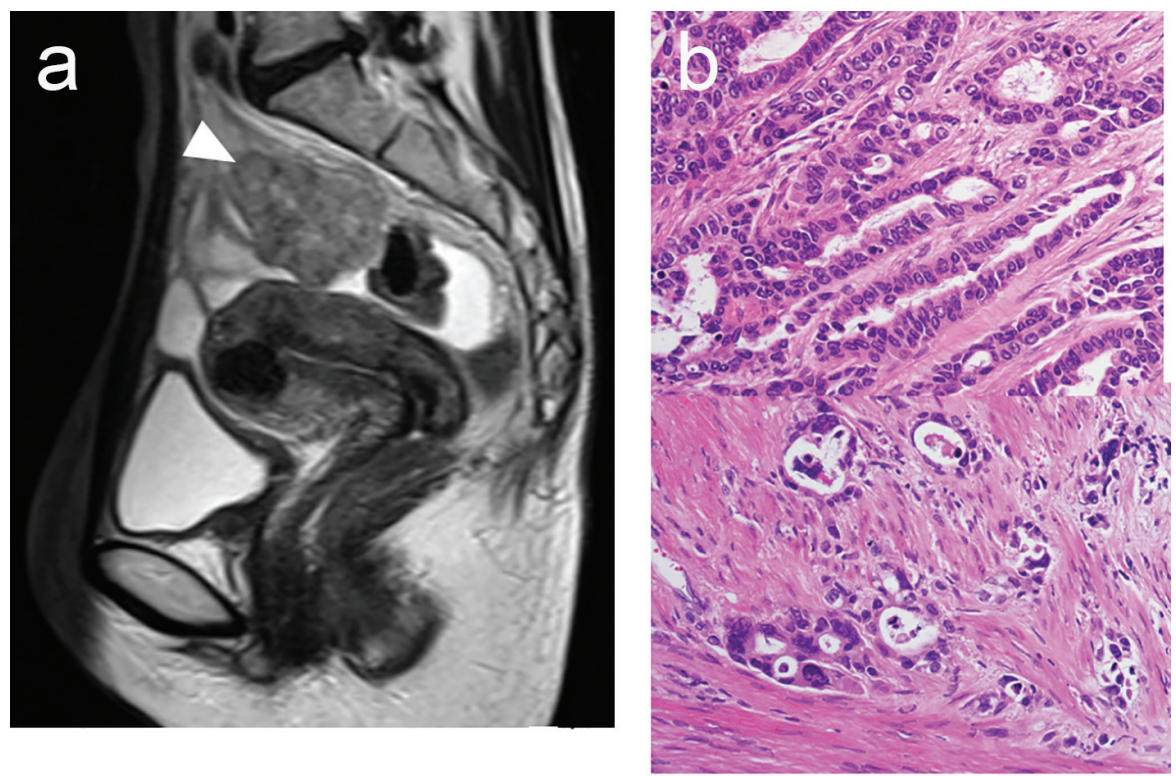

Figure 3. MRI in June 2012 revealed metastases in the right ovary and uterus (arrowhead) (a). Necropsy of the ovary metastasis demonstrated adenocarcinoma accompanied by trophoblastic differentiation (b). 
Table 1. The Cases of Lung Carcinoma With Trophoblastic Differentiation or Choriocarcinoma of the Lung

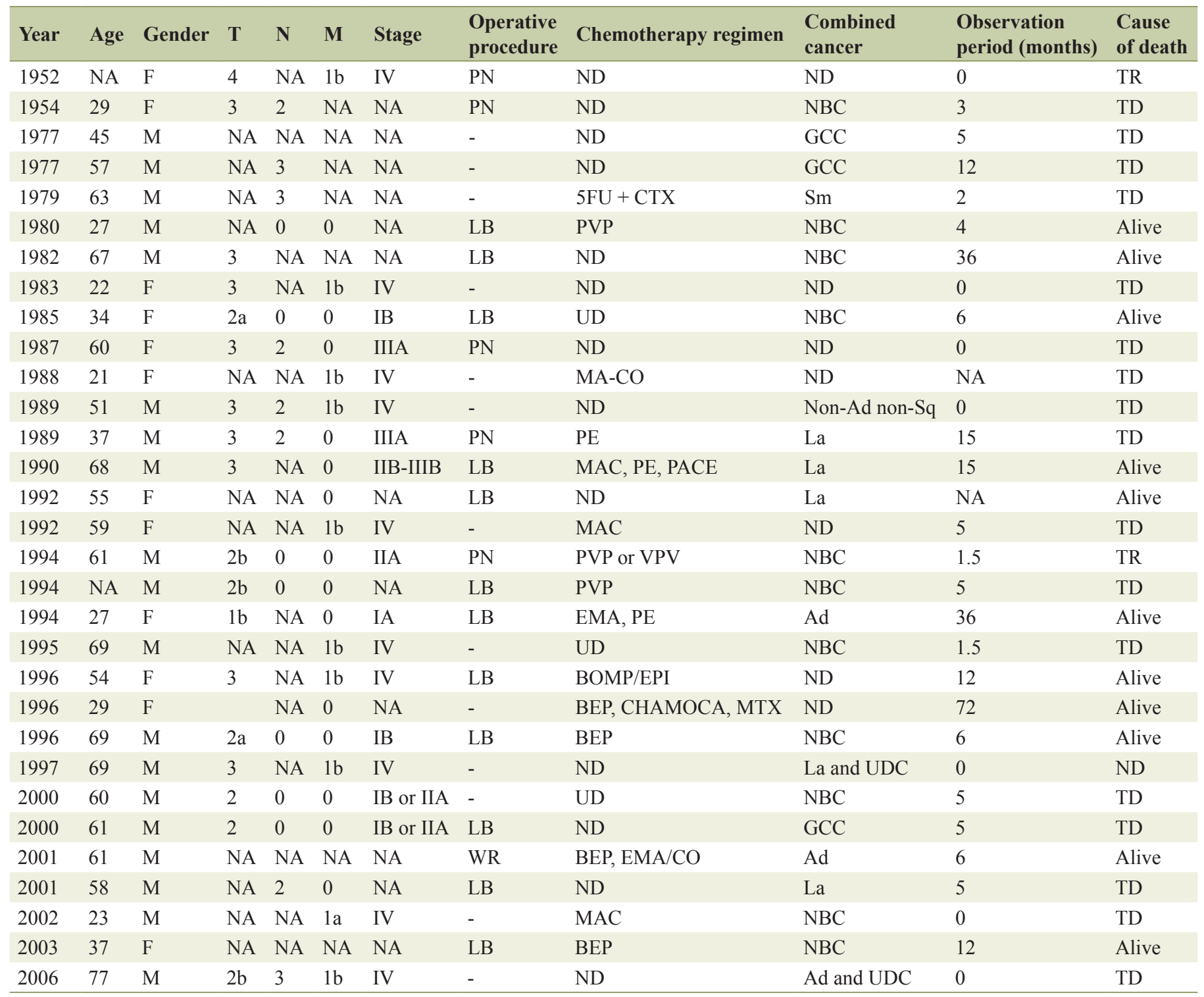

F: female; M: male; NA: not available; PN: pneumonectomy; LB: lobectomy; WR: wedge resection; ND: no description; 5FU: 5-flourouracil; CTX: cyclophosphamide; PVP: cisplatin and etoposide; MA-CO: methotrexate, actinomycin D/oncovin, cyclophosphamide; PE: etoposide and cisplatin; MAC: methotrexate/leucovorin, actinomycin-D, and cyclophosphamide or chlorambucil; PACE: cisplatin, adriamycin and cytoxan; VPV: bleomycin by vinblastine; EMA: etoposide and cisplatin/etoposide, methotrexate, and actinomycin D; BOMP-EPI: bleomycin, vincristine, methotrexate, platinum/ etoposide, platinum, ifosfamide; BEP: bleomycin, etoposide, cisplatin; CHAMOCA: cyclophosphamide, hydroxyurea, actinomycin D, methotrexate, doxorubicin, melphalan and vincristine; MTX: methotrexate; UD: unknown details; NBC: nothing but choriocarcinoma; GCC: giant cell carcinoma; Sm: small cell carcinoma; Ad: adenocarcinoma; Sq: squamous cell carcinoma; La: large cell carcinoma; UDC: undifferentiated carcinoma; TR: treatment-related death; TD: tumor death.

administrated. However, 2 months later, MRI demonstrated newly developed multiple brain metastases, and 30 Gy of whole-brain irradiation was performed. Despite these treatments, the serum level of hCG increased to $6.5 \mathrm{mIU} / \mathrm{mL}$. The L858R mutation of exon21 in the EGFR gene was confirmed, and gefitinib was administered at $250 \mathrm{mg}$ /day through June 2011 until June 2012 without any severe adverse reaction except for grade 2 dermatitis. By December 2011, the serum level of hCG had decreased to $4.1 \mathrm{mIU} / \mathrm{mL}$. In May 2012, the serum level of hCG was $4.3 \mathrm{mIU} / \mathrm{mL}$, and FDG-PET and abdominal MRI revealed newly developed metastases in the right ovary and uterus in June 2012 (Fig. 3a). Combination chemotherapy with cisplatin and pemetrexed was administered, but the second cycle had to be suspended due to grade 3 hyponatremia. Pemetrexed was then administered as a single agent, but hyponatremia and performance status 4 also disturbed the second cycle or any further anti-cancer therapy. The patient died in January 2013, and necropsy of the right 
ovarian metastasis revealed invasive proliferation of adenocarcinoma with trophoblastic differentiation and positivity for TTF-1 and hCG (Fig. 3b).

\section{Discussion}

The serum level of hCG was an accurate indicator of tumor aggravation which was $4.9,3.4,5.0$ and $4.1 \mathrm{mIU} / \mathrm{mL}$ in the preoperative period, during postoperative adjuvant chemotherapy, and when brain metastasis was first detected and gefitinib therapy initiated, respectively.

Complete resection and standard adjuvant chemotherapy resulted in a 10-month postoperative tumor-free period, and gefitinib therapy, believed to prolong the median progressionfree period approximately 9.5 months among the epidermal growth factor receptor mutant positive non-small lung cancer patients, achieved a 12-month progression-free period in this case after recurrence [1]. To our best knowledge, this is the first report to describe the effects of gefitinib for primary lung adenocarcinoma with trophoblastic differentiation.

In the latest WHO classification, the term primary choriocarcinoma of the lung was thought to be contradictory [2]. Table 1 lists 31 cases of lung carcinoma with trophoblastic differentiation, or the choriocarcinoma of the lung, retrieved from the English literature [3-8]. For 18 of the 31 cases, including duplicates, anti-cancer chemotherapy was employed using the regimens shown in Table 1. All of these regimens were regarded as the appropriate protocol for germ cell tumors, except for $\mathrm{PE}$ and PACE which are currently appropriate protocols for small cell carcinoma of the lung. The three patients who received PE or PACE survived for more than 15 months. The remaining 14 patients treated with protocols for germ cell tumor died within 12 months except one case. These results suggest that chemotherapy regimens for primary lung cancer other than germ cell tumor might be suitable for the lung carcinoma with trophoblastic differentiation.

\section{Conclusion}

We have reported a case of lung adenocarcinoma with trophoblastic differentiation. Complete resection and adjuvant chemotherapy achieved a 10-month tumor-free period, and use of gefitinib led to a 12-month progression-free period.

\section{Conflicts of Interest}

Yasumichi Yamamoto, Toshiya Toyazaki and Shinji Kosaka have no conflicts of interest.

\section{Grant or Financial Support}

None.

\section{References}

1. Mok TS, Wu YL, Thongprasert S, Yang CH, Chu DT, Saijo N, Sunpaweravong P, et al. Gefitinib or carboplatinpaclitaxel in pulmonary adenocarcinoma. N Engl J Med. 2009;361(10):947-957.

2. Nicholson AG, Teratoma IN, Travis WD. In: Brambilla E, Mueller-Hermelink HK, Harris CC, eds. World Health Organization Classification of Tumors 7. Pathology and genetics of tumor of the lung, pleura, thymus and heart. Lyon: IARC press; 2004: 119.

3. Tsai JR, Chong IW, Hung JY, Tsai KB. Use of urine pregnancy test for rapid diagnosis of primary pulmonary choriocarcinoma in a man. Chest. 2002;121(3):996-998.

4. Ikura $Y$, Inoue $T$, Tsukuda $H$, Yamamoto $T$, Ueda M, Kobayashi Y. Primary choriocarcinoma and human chorionic gonadotrophin-producing giant cell carcinoma of the lung: are they independent entities? Histopathology. 2000;36(1):17-25.

5. Chen F, Tatsumi A, Numoto S. Combined choriocarcinoma and adenocarcinoma of the lung occurring in a man: case report and review of the literature. Cancer. 2001;91(1):123-129.

6. Sawa H, Taomoto K, Tamaki N, Obayashi C, Nishimura R. Metastatic bronchogenic carcinoma with human chorionic gonadotropin production manifesting as cerebellar hemorrhage - case report. Neurol Med Chir (Tokyo). 2001;41(8):406-410.

7. Arslanian A, Pischedda F, Filosso PL, Di Marzio P, Oliaro A, Fraire F, Papotti M. Primary choriocarcinoma of the lung. J Thorac Cardiovasc Surg. 2003;125(1):193-196.

8. Yamamoto S, Tanaka H, Takeo H, Yasuda K, Mastukuma S. Primary pulmonary choriocarcinoma combined with adenocarcinoma. Pathol Int. 2006;56(7):402-407. 